\title{
Research Article \\ Strong Convergence of the Viscosity Approximation Process for the Split Common Fixed-Point Problem of Quasi-Nonexpansive Mappings
}

\author{
Jing Zhao and Songnian He \\ College of Science, Civil Aviation University of China, Tianjin 300300, China \\ Correspondence should be addressed to Jing Zhao, zhaojing200103@163.com \\ Received 14 December 2011; Accepted 11 January 2012 \\ Academic Editor: Yonghong Yao
}

Copyright (c) 2012 J. Zhao and S. He. This is an open access article distributed under the Creative Commons Attribution License, which permits unrestricted use, distribution, and reproduction in any medium, provided the original work is properly cited.

Very recently, Moudafi (2011) introduced an algorithm with weak convergence for the split common fixed-point problem. In this paper, we will continue to consider the split common fixedpoint problem. We discuss the strong convergence of the viscosity approximation method for solving the split common fixed-point problem for the class of quasi-nonexpansive mappings in Hilbert spaces. Our results improve and extend the corresponding results announced by many others.

\section{Introduction and Preliminary}

Throughout this paper, we always assume that $H$ is a real Hilbert space with inner product $\langle\cdot, \cdot\rangle$ and norm $\|\cdot\|$. Let $I$ denote the identity operator on $H$. Let $C$ and $Q$ be nonempty closed convex subset of real Hilbert spaces $H_{1}$ and $H_{2}$, respectively. The split feasibility problem (SFP) is to find a point

$$
x \in C \text { such that } A x \in Q \text {, }
$$

where $A: H_{1} \rightarrow H_{2}$ is a bounded linear operator. The SFP in finite-dimensional Hilbert spaces was first introduced by Censor and Elfving [1] for modeling inverse problems which arise from phase retrievals and in medical image reconstruction [2]. The SFP attracts many authors' attention due to its application in signal processing. Various algorithms have been invented to solve it (see [3-9] and references therein). 
Note that the split feasibility problem (1.1) can be formulated as a fixed-point equation by using the fact

$$
P_{C}\left(I-\gamma A^{*}\left(I-P_{Q}\right) A\right) x^{*}=x^{*}
$$

that is, $x^{*}$ solves the SFP (1.1) if and only if $x^{*}$ solves the fixed point equation (1.2) (see [10] for the details). This implies that we can use fixed-point algorithms (see [11-13]) to solve SFP. A popular algorithm that solves the SFP (1.1) is due to Byrne's CQ algorithm [2] which is found to be a gradient-projection method (GPM) in convex minimization. Subsequently, Byrne [3] applied KM iteration to the CQ algorithm, and Zhao and Yang [14] applied KM iteration to the perturbed CQ algorithm to solve the SFP. It is well known that the CQ algorithm and the KM algorithm for a split feasibility problem do not necessarily converge strongly in the infinite-dimensional Hilbert spaces.

The split common fixed-point problem (SCFP) is a generalization of the split feasibility problem (SFP) and the convex feasibility problem (CFP); see [15]. In this paper, we introduce and study the convergence properties of a viscosity approximation algorithm for solving the SCFP for the class of quasi-nonexpansive operators $S$ such that $I-S$ is demiclosed at the origin.

Now let us first recall the definition of quasi-nonexpansive operators which appear naturally when using subgradient projection operator techniques in solving some feasibility problems, and also some definitions of classes of operators often used in fixed-point theory and which are commonly encountered in the literature.

Let $T: H \rightarrow H$ be a mapping. A point $x \in H$ is said to be a fixed point of $T$ provided that $T x=x$. In this paper, we use $F(T)$ to denote the fixed-point set and use $\rightarrow$ and $\rightarrow$ to denote the strong convergence and weak convergence, respectively. We use $\omega_{w}\left(x_{k}\right)=\{x$ : $\left.\exists x_{k_{j}} \rightarrow x\right\}$ stand for the weak $\omega$-limit set of $\left\{x_{k}\right\}$.

(i) A mapping $T: H \rightarrow H$ belongs to the general class $\Phi_{Q}$ of (possibly discontinuous) quasi-nonexpansive mappings if

$$
\|T x-q\| \leq\|x-q\|, \quad \forall(x, q) \in H \times F(T) .
$$

(ii) A mapping $T: H \rightarrow H$ belongs to the set $\Phi_{N}$ of nonexpansive mappings if

$$
\|T x-T y\| \leq\|x-y\|, \quad \forall(x, y) \in H \times H .
$$

(iii) A mapping $T: H \rightarrow H$ belongs to the set $\Phi_{\mathrm{FN}}$ of firmly nonexpansive mappings if

$$
\|T x-T y\|^{2} \leq\|x-y\|^{2}-\|(x-y)-(T x-T y)\|^{2}, \quad \forall(x, y) \in H \times H .
$$

(iv) A mapping $T: H \rightarrow H$ belongs to the set $\Phi_{\mathrm{FQ}}$ of firmly quasi-nonexpansive mappings if

$$
\|T x-q\|^{2} \leq\|x-q\|^{2}-\|x-T x\|^{2}, \quad \forall(x, q) \in H \times F(T) .
$$


It is easily observed that $\Phi_{\mathrm{FN}} \subset \Phi_{N} \subset \Phi_{Q}$ and that $\Phi_{\mathrm{FN}} \subset \Phi_{\mathrm{FQ}} \subset \Phi_{Q}$. Furthermore, $\Phi_{\mathrm{FN}}$ is well known to include resolvents and projection operators, while $\Phi_{\mathrm{FQ}}$ contains subgradient projection operators (see, e.g., [16] and the reference therein).

A mapping $T: H \rightarrow H$ is called demiclosed at the origin if any sequence $\left\{x_{n}\right\}$ weakly converges to $x$, and if the sequence $\left\{T x_{n}\right\}$ strongly converges to 0 , then $T x=0$. A mapping $f: H \rightarrow H$ is called a contraction of modulus $\rho \in[0,1)$ if

$$
\|f x-f y\| \leq \rho\|x-y\|, \quad \forall(x, y) \in H \times H .
$$

In what follows, we will focus our attention on the following general two-operator split common fixed-point problem:

$$
\text { find } x^{*} \in C \text { such that } A x^{*} \in Q \text {, }
$$

where $A: H_{1} \rightarrow H_{2}$ is a bounded linear operator, $U: H_{1} \rightarrow H_{1}$ and $S: H_{2} \rightarrow H_{2}$ are two quasi-nonexpansive operators with nonempty fixed-point sets $F(U)=C$ and $F(S)=Q$, and denote the solution set of the two-operator SCFP by

$$
\Gamma=\{y \in C ; A y \in Q\} .
$$

Recall that $F(U)$ and $F(S)$ are nonempty closed convex subsets of $H_{1}$ and $H_{2}$, respectively. If $\Gamma \neq \emptyset$, we have $\Gamma$ which is close convex subset of $H_{1}$. To solve (1.8), Censor and Segal [15] proposed and proved, in infinite-dimensional spaces, the convergence of the following algorithm:

$$
x_{k+1}=U\left(x_{k}+\gamma A^{t}(S-I) A x_{k}\right), \quad k \in N,
$$

where $\gamma \in(0,2 / \lambda)$, with $\lambda$ being the largest eigenvalue of the matrix $A^{t} A$ ( $A^{t}$ stands for matrix transposition). Very recently, Moudafi [17] introduced the following relaxed algorithm:

$$
x_{k+1}=\left(1-\alpha_{k}\right) u_{k}+\alpha_{k} U\left(u_{k}\right), \quad k \in N,
$$

where $u_{k}=x_{k}+\gamma \beta A^{*}(S-I) A x_{k}, \beta \in(0,1), \alpha_{k} \in(0,1)$, and $\gamma \in(0,1 / \lambda \beta)$, with $\lambda$ being the spectral radius of the operator $A^{*} A$. Moudafi proved weak convergence result of the algorithm in Hilbert spaces.

Inspired by their work, we introduce the following viscosity approximation algorithm.

Algorithm 1. Initialization: Let $x_{0} \in H$ be arbitrary.

Iterative step: Set $T=U\left(I+\gamma A^{*}(S-I) A\right)$. For $k \in N$, let

$$
x_{k+1}=\alpha_{k} f\left(x_{k}\right)+\left(1-\alpha_{k}\right)\left(\left(1-\omega_{k}\right) x_{k}+\omega_{k} T x_{k}\right),
$$

where $f: H \rightarrow H$ is a contraction of modulus $\rho, \omega_{k} \in(0,1 / 2), \gamma \in(0,1 / \lambda)$ with $\lambda$ being the spectral radius of the operator $A^{*} A$, and $\alpha_{k} \in(0,1)$. 
This paper establishes the strong convergence of the sequence given by (1.12) to the unique solution of the variational inequality problem $\operatorname{VIP}(I-f, \Gamma)$ :

$$
\text { find } x^{*} \in \Gamma \quad \text { such that }\left\langle(I-f) x^{*}, v-x^{*}\right\rangle \geq 0, \quad \forall v \in \Gamma \text {. }
$$

Now we give a series of preliminary results needed for the convergence analysis of algorithm (1.12).

Lemma 1.1. Let $H$ be a real Hilbert space and $T: H \rightarrow H$ a quasi-nonexpansive mapping. Then, the following properties are reached:

(i) $\langle x, y\rangle=-\frac{1}{2}\|x-y\|^{2}+\frac{1}{2}\|x\|^{2}+\frac{1}{2}\|y\|^{2}, \quad \forall(x, y) \in H \times H$;

(ii) $\langle x-T x, x-q\rangle \geq \frac{1}{2}\|x-T x\|^{2}$ and $\langle x-T x, q-T x\rangle \leq \frac{1}{2}\|x-T x\|^{2}, \quad \forall(x, q) \in H \times F(T)$.

Remark 1.2. Let $F:=I-f$, where $f$ is the contraction defined in (1.7). It is a simple matter to see that the operator $F$ is $(1-\rho)$ strongly monotone over $H$; that is,

$$
\langle F x-F y, x-y\rangle \geq(1-\rho)\|x-y\|^{2}, \quad \forall(x, y) \in H \times H .
$$

The next result is of fundamental importance for the techniques of analysis used in this paper. It was established in [18], and its proof is given for the sake of completeness.

Lemma 1.3 (see [18, Lemma 1.3]). Let $\left\{\delta_{n}\right\}$ be a sequence of real numbers that does not decrease at infinity, in the sense that there exists a subsequence $\left\{\delta_{n_{j}}\right\}_{j \geq 0}$ of $\left\{\delta_{n}\right\}$ which satisfies $\delta_{n_{j}}<\delta_{n_{j}+1}$ for all $j \geq 0$. Also consider the sequence of integers $\{\tau(n)\}_{n \geq n_{0}}$ defined by

$$
\tau(n)=\max \left\{k \leq n \mid \delta_{k}<\delta_{k+1}\right\} .
$$

Then $\{\tau(n)\}_{n \geq n_{0}}$ is a nondecreasing sequence verifying $\lim _{n \rightarrow \infty} \tau(n)=\infty$, and, for all $n \geq n_{0}$, it holds that $\delta_{\tau(n)} \leq \delta_{\tau(n)+1}$ and one has

$$
\delta_{n} \leq \delta_{\tau(n)+1}
$$

Proof. Clearly, we can see that $\{\tau(n)\}$ is a well-defined sequence, and the fact that it is nondecreasing is obvious as well as $\lim _{n \rightarrow \infty} \tau(n)=\infty$ and $\delta_{\tau(n)} \leq \delta_{\tau(n)+1}$. Let us prove (1.16). It is easily observed that $\tau(n) \leq n$. Consequently, we prove (1.16) by distinguishing the three cases: (c1) $\tau(n)=n$; (c2) $\tau(n)=n-1$; (c3) $\tau(n)<n-1$. In the first case (i.e., $\tau(n)=n),(1.16)$ is immediately given by $\delta_{\tau(n)} \leq \delta_{\tau(n)+1}$. In the second case (i.e., $\left.\tau(n)=n-1\right)$, (1.16) becomes obvious. In the third case (i.e., $\tau(n) \leq n-2)$, by (1.15) and for any integer $n \geq n_{0}$, we easily observe that $\delta_{j} \geq \delta_{j+1}$ for $\tau(n)+1 \leq j \leq n-1$; namely,

$$
\delta_{\tau(n)+1} \geq \delta_{\tau(n)+2} \geq \cdots \geq \delta_{n-1} \geq \delta_{n}
$$

which entails the desired result. 


\section{Main Results}

Theorem 2.1. Given a bounded linear operator $A: H_{1} \rightarrow H_{2}$, let $U: H_{1} \rightarrow H_{1}$ and $S: H_{2} \rightarrow H_{2}$ be quasi-nonexpansive mappings with nonempty fixed-point set $F(U)=C$ and $F(S)=Q$. Assume that $U-I$ and $S-I$ are demiclosed at origin. Let $\left\{x_{k}\right\}$ be the sequence given by (1.12) with $\gamma \in$ $(0,1 / \lambda), \omega_{k} \in(0,1 / 2)$ such that $0<\liminf _{k \rightarrow \infty} \omega_{k} \leq \limsup _{k \rightarrow \infty} \omega_{k}<1 / 2$ and $\left\{\alpha_{k}\right\} \subset(0,1)$ such that $\lim _{k \rightarrow \infty} \alpha_{k}=0$ and $\sum_{k} \alpha_{k}=\infty$. If $\Gamma \neq \emptyset$, then the sequence $\left\{x_{k}\right\}$ strongly converges to a split common fixed-point $x^{*} \in \Gamma$, verifying $x^{*}=P_{\Gamma} f\left(x^{*}\right)$ which equivalently solves the following variational inequality problem:

$$
x^{*} \in \Gamma, \quad\left\langle(I-f) x^{*}, v-x^{*}\right\rangle \geq 0, \quad \forall v \in \Gamma .
$$

Proof. Set $T_{\omega_{k}}=\left(1-\omega_{k}\right) I+\omega_{k} T$. Then $x_{k+1}=\alpha_{k} f\left(x_{k}\right)+\left(1-\alpha_{k}\right) T_{\omega_{k}} x_{k}$.

Firstly, we prove that $\left\{x_{k}\right\}$ is bounded. Taking $y \in \Gamma$, that is, $y \in F(U), A y \in F(S)$. We have

$$
\begin{aligned}
\left\|x_{k+1}-y\right\| & =\left\|\alpha_{k}\left(f\left(x_{k}\right)-f(y)\right)+\alpha_{k}(f(y)-y)+\left(1-\alpha_{k}\right)\left(T_{\omega_{k}} x_{k}-y\right)\right\| \\
& \leq \alpha_{k}\left\|f\left(x_{k}\right)-f(y)\right\|+\alpha_{k}\|f(y)-y\|+\left(1-\alpha_{k}\right)\left\|T_{\omega_{k}} x_{k}-y\right\| \\
& \leq \alpha_{k} \rho\left\|x_{k}-y\right\|+\alpha_{k}\|f(y)-y\|+\left(1-\alpha_{k}\right)\left\|T_{\omega_{k}} x_{k}-y\right\| .
\end{aligned}
$$

From the definition of $T_{\omega_{k}}$, we get

$$
\begin{aligned}
\left\|T_{\omega_{k}} x_{k}-y\right\|^{2} & =\left\|\left(1-\omega_{k}\right) x_{k}+\omega_{k} T x_{k}-y\right\|^{2} \\
& =\left\|x_{k}-y+\omega_{k}\left(T x_{k}-x_{k}\right)\right\|^{2} \\
& =\left\|x_{k}-y\right\|^{2}-2 \omega_{k}\left\langle x_{k}-y, x_{k}-T x_{k}\right\rangle+\omega_{k}^{2}\left\|T x_{k}-x_{k}\right\|^{2} .
\end{aligned}
$$

On the other hand, we have

$$
\begin{aligned}
\left\|T x_{k}-y\right\|^{2} & =\left\|U\left(I+\gamma A^{*}(S-I) A\right) x_{k}-y\right\|^{2} \\
& \leq\left\|\left(I+\gamma A^{*}(S-I) A\right) x_{k}-y\right\|^{2} \\
& =\left\|x_{k}-y\right\|^{2}+\gamma^{2}\left\|A^{*}(S-I) A x_{k}\right\|^{2}+2 \gamma\left\langle x_{k}-y, A^{*}(S-I) A x_{k}\right\rangle \\
& =\left\|x_{k}-y\right\|^{2}+\gamma^{2}\left\langle(S-I) A x_{k}, A A^{*}(S-I) A x_{k}\right\rangle+2 \gamma\left\langle x_{k}-y, A^{*}(S-I) A x_{k}\right\rangle .
\end{aligned}
$$

From the definition of $\lambda$, it follows that

$$
\begin{aligned}
\gamma^{2}\left\langle(S-I) A x_{k}, A A^{*}(S-I) A x_{k}\right\rangle & \leq \lambda \gamma^{2}\left\langle(S-I) A x_{k},(S-I) A x_{k}\right\rangle \\
& =\lambda \gamma^{2}\left\|(S-I) A x_{k}\right\|^{2} .
\end{aligned}
$$


Now, by using property (ii) of Lemma 1.1, we obtain

$$
\begin{aligned}
2 \gamma\left\langle x_{k}-y, A^{*}(S-I) A x_{k}\right\rangle & =2 \gamma\left\langle A\left(x_{k}-y\right),(S-I) A x_{k}\right\rangle \\
& =2 \gamma\left\langle A\left(x_{k}-y\right)+(S-I) A x_{k}-(S-I) A x_{k},(S-I) A x_{k}\right\rangle \\
& =2 \gamma\left(\left\langle S\left(A x_{k}\right)-A y_{,}(S-I) A x_{k}\right\rangle-\left\|(S-I) A x_{k}\right\|^{2}\right) \\
& \leq 2 \gamma\left(\frac{1}{2}\left\|(S-I) A x_{k}\right\|^{2}-\left\|(S-I) A x_{k}\right\|^{2}\right) \\
& =-\gamma\left\|(S-I) A x_{k}\right\|^{2} .
\end{aligned}
$$

Combining (2.4)-(2.6), we have

$$
\begin{aligned}
\left\|T x_{k}-y\right\|^{2} & \leq\left\|x_{k}-y\right\|^{2}+\lambda \gamma^{2}\left\|(S-I) A x_{k}\right\|^{2}-\gamma\left\|(S-I) A x_{k}\right\|^{2} \\
& =\left\|x_{k}-y\right\|^{2}-\gamma(1-\lambda \gamma)\left\|(S-I) A x_{k}\right\|^{2} \\
& \leq\left\|x_{k}-y\right\|^{2} .
\end{aligned}
$$

From property (i) of Lemma 1.1, we have

$$
\begin{aligned}
\left\langle x_{k}-y, x_{k}-T x_{k}\right\rangle & =-\frac{1}{2}\left\|T x_{k}-y\right\|^{2}+\frac{1}{2}\left\|x_{k}-y\right\|^{2}+\frac{1}{2}\left\|x_{k}-T x_{k}\right\|^{2} \\
& \geq \frac{1}{2}\left\|x_{k}-T x_{k}\right\|^{2} .
\end{aligned}
$$

From (2.3) and (2.8), we have

$$
\begin{aligned}
\left\|T_{\omega_{k}} x_{k}-y\right\|^{2} & \leq\left\|x_{k}-y\right\|^{2}-\omega_{k}\left\|x_{k}-T x_{k}\right\|^{2}+\omega_{k}^{2}\left\|x_{k}-T x_{k}\right\|^{2} \\
& =\left\|x_{k}-y\right\|^{2}-\omega_{k}\left(1-\omega_{k}\right)\left\|x_{k}-T x_{k}\right\|^{2} \\
& \leq\left\|x_{k}-y\right\|^{2}
\end{aligned}
$$

Combining (2.2), (2.3), and (2.9), it follows that

$$
\begin{aligned}
\left\|x_{k+1}-y\right\| & \leq \alpha_{k} \rho\left\|x_{k}-y\right\|+\alpha_{k}\|f(y)-y\|+\left(1-\alpha_{k}\right)\left\|x_{k}-y\right\| \\
& =\left[1-\alpha_{k}(1-\rho)\right]\left\|x_{k}-y\right\|+\alpha_{k}\|f(y)-y\| \\
& \leq \max \left\{\left\|x_{k}-y\right\|, \frac{1}{1-\rho}\|f(y)-y\|\right\} .
\end{aligned}
$$


It is obviously that

$$
\left\|x_{k}-y\right\| \leq \max \left\{\left\|x_{0}-y\right\|, \frac{1}{1-\rho}\|f(y)-y\|\right\}
$$

and hence $\left\{x_{k}\right\}$ is bounded. Let $x^{*}=P_{\Gamma} f\left(x^{*}\right)$. We have

$$
x_{k+1}-x_{k}+\alpha_{k}\left(x_{k}-f\left(x_{k}\right)\right)=\left(1-\alpha_{k}\right)\left(T_{\omega_{k}} x_{k}-x_{k}\right),
$$

and hence

$$
\left\langle x_{k+1}-x_{k}+\alpha_{k}(I-f) x_{k}, x_{k}-x^{*}\right\rangle=-\left(1-\alpha_{k}\right)\left\langle x_{k}-T_{\omega_{k}} x_{k}, x_{k}-x^{*}\right\rangle .
$$

By (2.9) we obtain that

$$
\begin{aligned}
\left\langle x_{k}-T_{\omega_{k}} x_{k}, x_{k}-x^{*}\right\rangle & =\frac{1}{2}\left\|x_{k}-T_{\omega_{k}} x_{k}\right\|^{2}+\frac{1}{2}\left\|x_{k}-x^{*}\right\|^{2}-\frac{1}{2}\left\|T_{\omega_{k}} x_{k}-x^{*}\right\|^{2} \\
& \geq \frac{\omega_{k}^{2}}{2}\left\|x_{k}-T x_{k}\right\|^{2}+\frac{1}{2}\left\|x_{k}-x^{*}\right\|^{2}-\frac{1}{2}\left\|x_{k}-x^{*}\right\|^{2}+\frac{\omega_{k}}{2}\left(1-\omega_{k}\right)\left\|x_{k}-T x_{k}\right\|^{2} \\
& =\frac{\omega_{k}}{2}\left\|x_{k}-T x_{k}\right\|^{2} .
\end{aligned}
$$

It follows from (2.13) that

$$
\left\langle x_{k+1}-x_{k}+\alpha_{k}(I-f) x_{k}, x_{k}-x^{*}\right\rangle \leq-\frac{\omega_{k}}{2}\left(1-\alpha_{k}\right)\left\|x_{k}-T x_{k}\right\|^{2},
$$

and hence

$$
-\left\langle x_{k}-x_{k+1}, x_{k}-x^{*}\right\rangle \leq-\alpha_{k}\left\langle(I-f) x_{k}, x_{k}-x^{*}\right\rangle-\frac{\omega_{k}}{2}\left(1-\alpha_{k}\right)\left\|x_{k}-T x_{k}\right\|^{2} .
$$

Setting $\delta_{k}=\frac{1}{2}\left\|x_{k}-x^{*}\right\|^{2}$, we have

$$
\begin{aligned}
\left\langle x_{k}-x_{k+1}, x_{k}-x^{*}\right\rangle & =-\frac{1}{2}\left\|x_{k+1}-x^{*}\right\|^{2}+\frac{1}{2}\left\|x_{k}-x^{*}\right\|^{2}+\frac{1}{2}\left\|x_{k}-x_{k+1}\right\|^{2} \\
& =-\delta_{k+1}+\delta_{k}+\frac{1}{2}\left\|x_{k}-x_{k+1}\right\|^{2}
\end{aligned}
$$

so that (2.16) can be rewritten as

$$
\delta_{k+1}-\delta_{k}-\frac{1}{2}\left\|x_{k}-x_{k+1}\right\|^{2} \leq-\alpha_{k}\left\langle(I-f) x_{k}, x_{k}-x^{*}\right\rangle-\frac{\omega_{k}}{2}\left(1-\alpha_{k}\right)\left\|x_{k}-T x_{k}\right\|^{2} .
$$


Now using (2.12) again, we have

$$
\begin{aligned}
\left\|x_{k+1}-x_{k}\right\|^{2} & =\| \alpha_{k}\left(f\left(x_{k}\right)-x_{k}\right)+\left(1-\alpha_{k}\right)\left(T_{\omega_{k}} x_{k}-x_{k} \|^{2}\right. \\
& \leq\left(\alpha_{k}\left\|f\left(x_{k}\right)-x_{k}\right\|+\left(1-\alpha_{k}\right)\left\|T_{\omega_{k}} x_{k}-x_{k}\right\|\right)^{2} \\
& \leq 2 \alpha_{k}^{2}\left\|f\left(x_{k}\right)-x_{k}\right\|^{2}+2\left(1-\alpha_{k}\right)^{2}\left\|T_{\omega_{k}} x_{k}-x_{k}\right\|^{2} \\
& \leq 2 \alpha_{k}^{2}\left\|f\left(x_{k}\right)-x_{k}\right\|^{2}+2\left(1-\alpha_{k}\right) \omega_{k}^{2}\left\|T x_{k}-x_{k}\right\|^{2},
\end{aligned}
$$

which yields

$$
\frac{1}{2}\left\|x_{k+1}-x_{k}\right\|^{2} \leq \alpha_{k}^{2}\left\|f\left(x_{k}\right)-x_{k}\right\|^{2}+\left(1-\alpha_{k}\right) \omega_{k}^{2}\left\|T x_{k}-x_{k}\right\|^{2}
$$

From (2.18) and (2.20), we obtain

$$
\delta_{k+1}-\delta_{k}+\omega_{k}\left(1-\alpha_{k}\right)\left(\frac{1}{2}-\omega_{k}\right)\left\|T x_{k}-x_{k}\right\|^{2} \leq \alpha_{k}\left[\alpha_{k}\left\|f\left(x_{k}\right)-x_{k}\right\|^{2}-\left\langle(I-f) x_{k}, x_{k}-x^{*}\right\rangle\right] .
$$

It follows from Remark 1.2 that

$$
\left\langle(I-f) x_{k}-(I-f) x^{*}, x_{k}-x^{*}\right\rangle \geq(1-\rho)\left\|x_{k}-x^{*}\right\|^{2}=2(1-\rho) \delta_{k}
$$

and hence

$$
2(1-\rho) \delta_{k}+\left\langle(I-f) x^{*}, x_{k}-x^{*}\right\rangle \leq\left\langle(I-f) x_{k}, x_{k}-x^{*}\right\rangle
$$

The rest of the proof will be divided into two parts.

Case 1. Suppose that there exists $k_{0}$ such that $\left\{\delta_{k}\right\}_{k \geq k_{0}}$ is nonincreasing. In this situation, $\left\{\delta_{k}\right\}$ is convergent because it is nonnegative, so that $\lim _{k \rightarrow \infty}\left(\delta_{k+1}-\delta_{k}\right)=0$; hence, in light of (2.21) together with $\alpha_{k} \rightarrow 0$, the boundedness of $\left\{x_{k}\right\}$, and $0<\liminf _{k \rightarrow \infty} \omega_{k} \leq \lim \sup _{k \rightarrow \infty} \omega_{k}<$ $1 / 2$, we obtain

$$
\lim _{k \rightarrow \infty}\left\|x_{k}-T x_{k}\right\|=0
$$

From (2.21) again, we have

$$
\alpha_{k}\left[-\alpha_{k}\left\|f\left(x_{k}\right)-x_{k}\right\|^{2}+\left\langle(I-f) x_{k}, x_{k}-x^{*}\right\rangle\right] \leq \delta_{k}-\delta_{k+1} .
$$

By $\sum_{k} \alpha_{k}=\infty$, we deduce that

$$
\liminf _{k \rightarrow \infty}\left(-\alpha_{k}\left\|f\left(x_{k}\right)-x_{k}\right\|^{2}+\left\langle(I-f) x_{k}, x_{k}-x^{*}\right\rangle\right) \leq 0
$$


and hence (as $\left.\alpha_{k}\left\|f\left(x_{k}\right)-x_{k}\right\|^{2} \rightarrow 0\right)$

$$
\liminf _{k \rightarrow \infty}\left\langle(I-f) x_{k}, x_{k}-x^{*}\right\rangle \leq 0
$$

By (2.23) and (2.27), we have

$$
\liminf _{k \rightarrow \infty}\left(2(1-\rho) \delta_{k}+\left\langle(I-f) x^{*}, x_{k}-x^{*}\right\rangle\right) \leq 0
$$

recalling that $\lim _{k \rightarrow \infty} \delta_{k}$ exists, we obtain

$$
2(1-\rho) \lim _{k \rightarrow \infty} \delta_{k}+\liminf _{k \rightarrow \infty}\left\langle(I-f) x^{*}, x_{k}-x^{*}\right\rangle \leq 0
$$

Now we prove that

$$
\liminf _{k \rightarrow \infty}\left\langle(I-f) x^{*}, x_{k}-x^{*}\right\rangle \geq 0
$$

It follows from (2.7) and (2.24) that

$$
\begin{aligned}
r(1-\lambda \gamma)\left\|(S-I) A x_{k}\right\|^{2} & \leq\left\|x_{k}-y\right\|^{2}-\left\|T x_{k}-y\right\|^{2} \\
& =\left(\left\|x_{k}-y\right\|-\left\|T x_{k}-y\right\|\right)\left(\left\|x_{k}-y\right\|+\left\|T x_{k}-y\right\|\right) \\
& \leq\left\|x_{k}-T x_{k}\right\|\left(\left\|x_{k}-y\right\|+\left\|T x_{k}-y\right\|\right) \\
& \longrightarrow 0 \quad(k \longrightarrow \infty)
\end{aligned}
$$

and hence

$$
\lim _{k \rightarrow \infty}\left\|(S-I) A x_{k}\right\|=0
$$

Taking $y \in \omega_{w}\left(x_{k}\right)$, from the demiclosedness of $S-I$ at 0 , we obtain

$$
S(A y)=A y
$$

Now, by setting $u_{k}=x_{k}+\gamma A^{*}(S-I) A x_{k}$, it follows that $y \in \omega_{w}\left(u_{k}\right)$. On the other hand,

$$
\left\|U\left(u_{k}\right)-u_{k}\right\|=\left\|T x_{k}-x_{k}-\gamma A^{*}(S-I) A x_{k}\right\| \leq\left\|T x_{k}-x_{k}\right\|+\gamma\left\|A^{*}\right\| \cdot\left\|(S-I) A x_{k}\right\| \longrightarrow 0
$$

which, combined with the demiclosedness of $U-I$ at 0 , yields

$$
U y=y
$$


Hence, $y \in C$ and $y \in \Gamma$. We can take subsequence $\left\{x_{k_{j}}\right\}$ of $\left\{x_{k}\right\}$ such that $x_{k_{j}} \rightarrow y$ as $\mathrm{j} \rightarrow \infty$ and

$$
\liminf _{k \rightarrow \infty}\left\langle(I-f) x^{*}, x_{k}-x^{*}\right\rangle=\lim _{j \rightarrow \infty}\left\langle(I-f) x^{*}, x_{k_{j}}-x^{*}\right\rangle
$$

which leads to

$$
\liminf _{k \rightarrow \infty}\left\langle(I-f) x^{*}, x_{k}-x^{*}\right\rangle=\left\langle(I-f) x^{*}, y-x^{*}\right\rangle \geq 0 .
$$

By (2.29), we have $\lim _{k \rightarrow \infty} \delta_{k}=0$, and hence $\left\{x_{k}\right\}$ converges strongly to $x^{*}$.

Case 2. Suppose there exists a subsequence $\left\{\delta_{k_{j}}\right\}_{j \geq 0}$ of $\left\{\delta_{k}\right\}$ such that $\delta_{k_{j}}<\delta_{k_{j}+1}$ for all $j \geq 0$. In this situation, we consider the sequence of indices $\{\tau(k)\}$ as defined in Lemma 1.3. It follows that $\delta_{\tau(k)+1}-\delta_{\tau(k)}>0$, which by (2.21) amounts to

$$
\begin{array}{r}
\omega_{k}\left(1-\alpha_{\tau(k)}\right)\left(\frac{1}{2}-\omega_{k}\right)\left\|T x_{\tau(k)}-x_{\tau(k)}\right\|^{2} \leq \alpha_{\tau(k)}[ \\
\alpha_{\tau(k)}\left\|f\left(x_{\tau(k)}\right)-x_{\tau(k)}\right\|^{2} \\
\left.-\left\langle(I-f) x_{\tau(k)}, x_{\tau(k)}-x^{*}\right\rangle\right] .
\end{array}
$$

By the boundedness of $\left\{x_{k}\right\}$ and $\alpha_{k} \rightarrow 0$, we immediately obtain

$$
\lim _{k \rightarrow \infty}\left\|T x_{\tau(k)}-x_{\tau(k)}\right\|=0
$$

Similar to Case 1, we have

$$
\liminf _{k \rightarrow \infty}\left\langle(I-f) x^{*}, x_{\tau(k)}-x^{*}\right\rangle \geq 0
$$

It follows from (2.38) that

$$
\left\langle(I-f) x_{\tau(k)}, x_{\tau(k)}-x^{*}\right\rangle \leq \alpha_{\tau(k)}\left\|f\left(x_{\tau(k)}\right)-x_{\tau(k)}\right\|^{2},
$$

which in the light of (2.23) yields

$$
2(1-\rho) \delta_{\tau(k)}+\left\langle(I-f) x^{*}, x_{\tau(k)}-x^{*}\right\rangle \leq \alpha_{\tau(k)}\left\|f\left(x_{\tau(k)}\right)-x_{\tau(k)}\right\|^{2}
$$

hence (as $\left.\alpha_{\tau(k)}\left\|f\left(x_{\tau(k)}\right)-x_{\tau(k)}\right\|^{2} \rightarrow 0\right)$ it follows that

$$
2(1-\rho) \limsup _{k \rightarrow \infty} \delta_{\tau(k)} \leq-\liminf _{k \rightarrow \infty}\left\langle(I-f) x^{*}, x_{\tau(k)}-x^{*}\right\rangle .
$$


From (2.40) we have $\lim \sup _{k \rightarrow \infty} \delta_{\tau(k)}=0$, so that $\lim _{k \rightarrow \infty} \delta_{\tau(k)}=0$, and hence $\lim _{k \rightarrow \infty} \| x_{\tau(k)}-$ $x^{*} \|=0$. On the other hand, it follows that

$$
\begin{aligned}
\left\|x_{\tau(k)+1}-x_{\tau(k)}\right\| & =\left\|\alpha_{\tau(k)}\left(f\left(x_{\tau(k)}\right)-x_{\tau(k)}\right)+\left(1-\alpha_{\tau(k)}\right)\left(T_{\omega_{k}} x_{\tau(k)}-x_{\tau(k)}\right)\right\| \\
& \leq \alpha_{\tau(k)}\left\|f\left(x_{\tau(k)}\right)-x_{\tau(k)}\right\|+\left(1-\alpha_{\tau(k)}\right) \omega_{k}\left\|T x_{\tau(k)}-x_{\tau(k)}\right\|,
\end{aligned}
$$

which, by (2.39), implies that

$$
\lim _{k \rightarrow \infty}\left\|x_{\tau(k)+1}-x_{\tau(k)}\right\|=0
$$

So we have

$$
\lim _{k \rightarrow \infty} \delta_{\tau(k)+1}=\frac{1}{2}\left\|x_{\tau(k)+1}-x^{*}\right\|=0
$$

Then, recalling that $\delta_{k} \leq \delta_{\tau(k)+1}$ (by Lemma 1.3), we get $\lim _{k \rightarrow \infty} \delta_{k}=0$, so that the sequence $\left\{x_{k}\right\}$ converges strongly to $x^{*}$.

Theorem 2.2. Given a bounded linear operator $A: H_{1} \rightarrow H_{2}$, let $U: H_{1} \rightarrow H_{1}$ and $S: H_{2} \rightarrow H_{2}$ be quasi-nonexpansive mappings with nonempty fixed-point set $F(U)=C$ and $F(S)=Q$. Assume that $U-I$ and $S-I$ are demiclosed at origin. Let $x_{0} \in H$ be arbitrary and $\left\{x_{k}\right\}$ the sequence given by

$$
x_{k+1}=\alpha_{k} f\left(x_{k}\right)+\left(1-\alpha_{k}\right)\left((1-\omega) x_{k}+\omega T x_{k}\right),
$$

where $T=U\left(I+\gamma A^{*}(S-I) A\right), f: H \rightarrow H$ a contraction of modulus $\rho, \gamma \in(0,1 / \lambda), \omega \in(0,1 / 2)$, and $\left\{\alpha_{k}\right\} \subset(0,1)$ such that $\lim _{k \rightarrow \infty} \alpha_{k}=0$ and $\sum_{k} \alpha_{k}=\infty$. If $\Gamma \neq \emptyset$, then the sequence $\left\{x_{k}\right\}$ strongly converges to a split common fixed-point $x^{*} \in \Gamma$, verifying $x^{*}=P_{\Gamma} f\left(x^{*}\right)$ which equivalently solves the following variational inequality problem:

$$
x^{*} \in \Gamma, \quad\left\langle(I-f) x^{*}, v-x^{*}\right\rangle \geq 0, \quad \forall v \in \Gamma .
$$

\section{Acknowledgments}

The research was supported by Fundamental Research Funds for the Central Universities (Program No. ZXH2011D005); it was also supported by science research foundation program in Civil Aviation University of China (2011kys02).

\section{References}

[1] Y. Censor and T. Elfving, "A multiprojection algorithm using Bregman projections in a product space," Numerical Algorithms, vol. 8, no. 2-4, pp. 221-239, 1994.

[2] C. Byrne, "Iterative oblique projection onto convex sets and the split feasibility problem," Inverse Problems, vol. 18, no. 2, pp. 441-453, 2002.

[3] C. Byrne, "A unified treatment of some iterative algorithms in signal processing and image reconstruction," Inverse Problems, vol. 20, no. 1, pp. 103-120, 2004. 
[4] P.-E. Maingé, "The viscosity approximation process for quasi-nonexpansive mappings in Hilbert spaces," Computers E Mathematics with Applications, vol. 59, no. 1, pp. 74-79, 2010.

[5] B. Qu and N. Xiu, "A note on the CQ algorithm for the split feasibility problem," Inverse Problems, vol. 21, no. 5, pp. 1655-1665, 2005.

[6] H.-K. Xu, "A variable Krasnosel'skii-Mann algorithm and the multiple-set split feasibility problem," Inverse Problems, vol. 22, no. 6, pp. 2021-2034, 2006.

[7] Q. Yang, "The relaxed CQ algorithm solving the split feasibility problem," Inverse Problems, vol. 20, no. 4, pp. 1261-1266, 2004.

[8] Q. Yang and J. Zhao, "Generalized KM theorems and their applications," Inverse Problems, vol. 22, no. 3, pp. 833-844, 2006.

[9] Y. Yao, W. Jigang, and Y.-C. Liou, "Regularized methods for the split feasibility problem," Abstract and Applied Analysis, vol. 2012, Article ID 140679, 13 pages, 2012.

[10] H.-K. Xu, "Iterative methods for the split feasibility problem in infinite-dimensional Hilbert spaces," Inverse Problems, vol. 26, no. 10, Article ID 105018, 2010.

[11] Y. Yao, Y.-C. Liou, and S. M. Kang, "Two-step projection methods for a system of variational inequality problems in Banach spaces," Journal of Global Optimization. In press.

[12] Y. Yao, R. Chen, and Y.-C. Liou, "A unified implicit algorithm for solving the triple-hierarchical constrained optimization problem," Mathematical and Computer Modelling, vol. 55, no. 3-4, pp. 15061515, 2012.

[13] Y. Yao, Y.-J. Cho, and Y.-C. Liou, "Hierarchical convergence of an implicit doublenet algorithm for nonexpansive semigroups and variational inequalities," Fixed Point Theory and Applications, vol. 2011, article 101, 2011.

[14] J. Zhao and Q. Yang, "Several solution methods for the split feasibility problem," Inverse Problems, vol. 21, no. 5, pp. 1791-1799, 2005.

[15] Y. Censor and A. Segal, "The split common fixed point problem for directed operators," Journal of Convex Analysis, vol. 16, no. 2, pp. 587-600, 2009.

[16] S. Măruşter and C. Popirlan, "On the Mann-type iteration and the convex feasibility problem," Journal of Computational and Applied Mathematics, vol. 212, no. 2, pp. 390-396, 2008.

[17] A. Moudafi, "A note on the split common fixed-point problem for quasi-nonexpansive operators," Nonlinear Analysis, vol. 74, no. 12, pp. 4083-4087, 2011.

[18] P.-E. Maingé, "Strong convergence of projected subgradient methods for nonsmooth and nonstrictly convex minimization," Set-Valued Analysis, vol. 16, no. 7-8, pp. 899-912, 2008. 


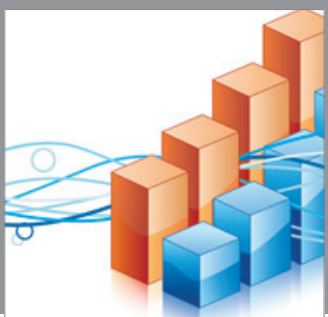

Advances in

Operations Research

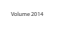

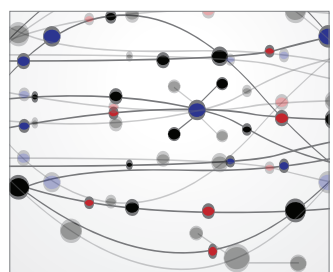

\section{The Scientific} World Journal
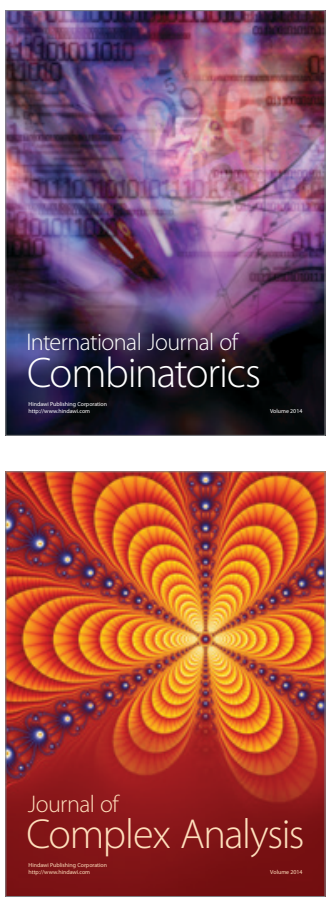

International Journal of

Mathematics and

Mathematical

Sciences
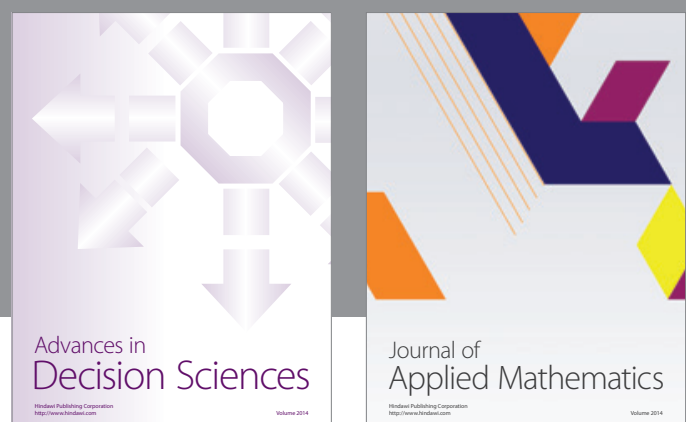

Journal of

Applied Mathematics
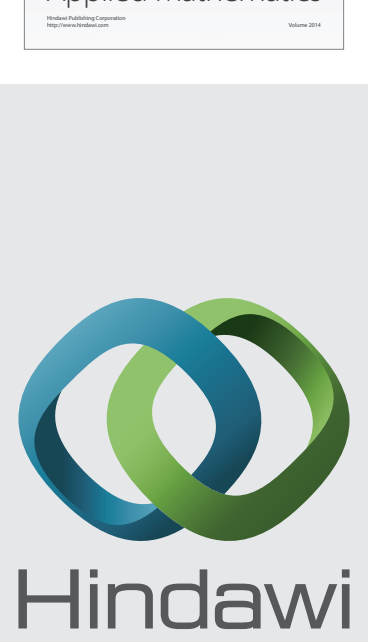

Submit your manuscripts at http://www.hindawi.com
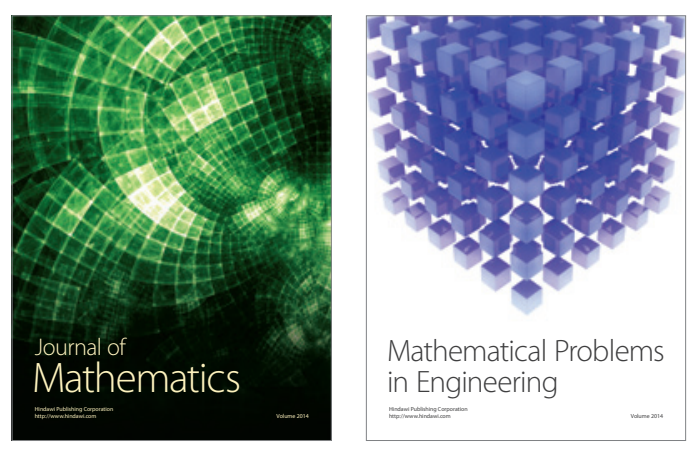

Mathematical Problems in Engineering
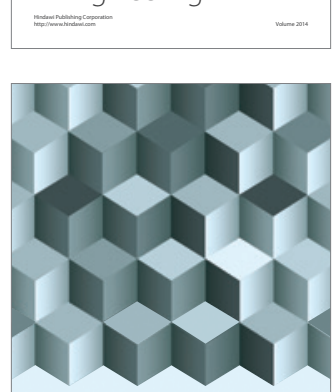

Journal of

Function Spaces
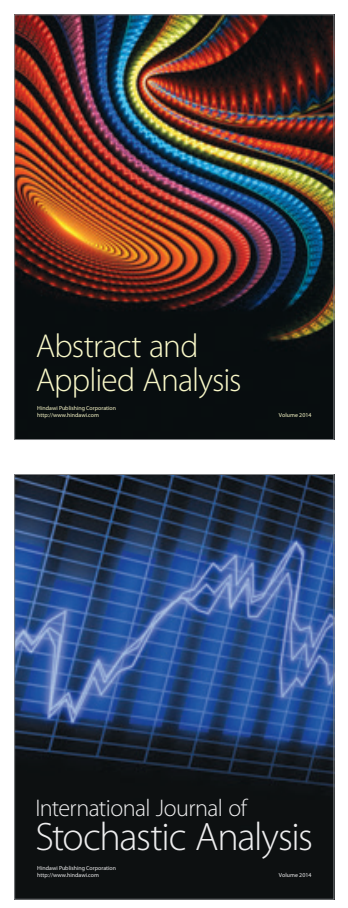

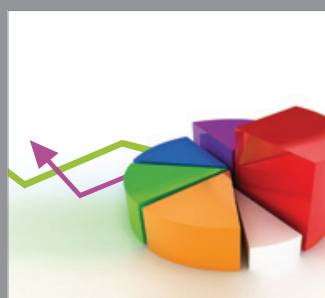

ournal of

Probability and Statistics

Promensencen
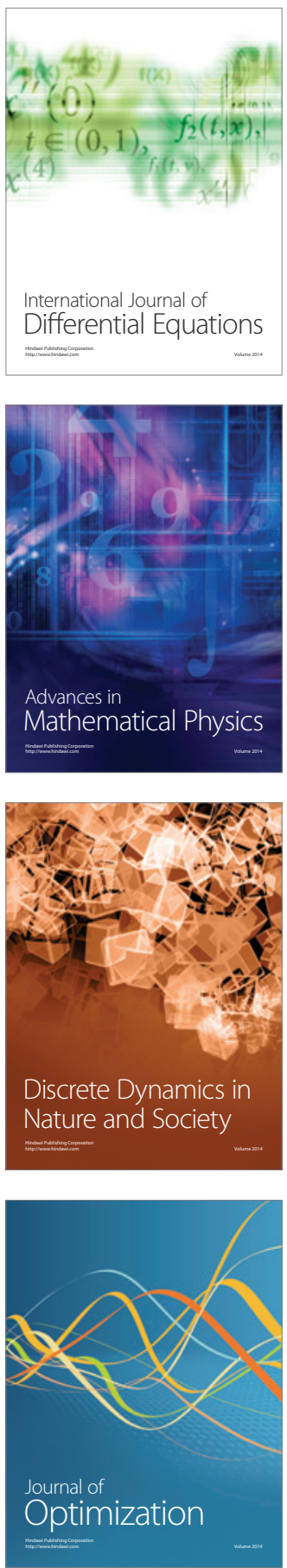